DESY 12-028

\title{
On Stability of the Electroweak Vacuum and the Higgs Portal
}

\author{
Oleg Lebedev \\ DESY Theory Group, Notkestrasse 85, D-22607 Hamburg, Germany
}

\begin{abstract}
In the Standard Model (SM), the Higgs mass around $125 \mathrm{GeV}$ implies that the electroweak vacuum is metastable since the quartic Higgs coupling turns negative at high energies. I point out that an arbitrarily small mixing of the Higgs with a heavy singlet can make the electroweak vacuum completely stable. This is due to a tree level correction to the Higgs mass, which survives in the zero-mixing/heavy-singlet limit. Such a situation is experimentally indistinguishable from the SM, unless the Higgs self-coupling can be measured. As a result, Higgs inflation and its variants can still be viable.
\end{abstract}




\section{Introduction}

The Higgs sector of the Standard Model (SM) enjoys a special status. The Higgs bilinear $H^{\dagger} H$ is the only gauge and Lorentz invariant dimension-2 operator that can be constructed out of the SM fields. This operator can couple to the "hidden" sector at the renormalizable level [1, 2],

$$
\Delta \mathcal{L}=c H^{\dagger} H S^{2}
$$

where $S$ is an SM singlet. More general allowed couplings include, for example, a coupling to massive vectors [3, 4] and a scalar curvature [5], which could be relevant to dark matter and inflation, respectively. In the case of a scalar Higgs portal, the phenomenology depends crucially on whether or not $S$ develops a vacuum expectation value (VEV). If it does, the Higgs boson mixes with the singlet [6, 7], otherwise $S$ becomes a good dak matter candidate [1, 8, 9]. Here we focus on the first possibility. A significant Higgs-singlet mixing can be probed at the LHC [10, 11] by measuring production cross sections for the Higgs-like states, whereas the small mixing $(<10 \%)$ case is much more challenging.

In this paper, we explore some consequences of an unobservably small Higgs-singlet mixing. We find, in particular, that such a mixing together with tiny singlet couplings can stabilize the electroweak (EW) vacuum, which otherwise appears metastable [12, 13]. Furthermore, it can lead to a significant $(\mathcal{O}(1))$ tree-level modification of the Higgs self-coupling, which can be measured at colliders. Finally, although Higgs inflation within the SM is disfavored by the tentative $125 \mathrm{GeV}$ Higgs signal seen at the LHC [14, 15], such a possibility remains open in our framework.

\section{Higgs portal potential and stability}

We start by reviewing properties of the Higgs portal potential following Ref. [16]. Related work can be found in [17, 18, 19].

\section{$2.1 \quad$ Relevant formulae}

The scalar potential in the unitary gauge $H^{T}=(0, h / \sqrt{2})$ is given by

$$
V=\frac{1}{4} \lambda_{h} h^{4}+\frac{1}{4} \lambda_{h s} s^{2} h^{2}+\frac{1}{4} \lambda_{s} s^{4}+\frac{1}{2} m_{h}^{2} h^{2}+\frac{1}{2} m_{s}^{2} s^{2} .
$$

Here $h$ and $s$ are real fields; $\lambda_{i}$ are the quartic couplings and $m_{i}^{2}$ are mass parameters. In what follows, we will be interested in the case when both $h$ and $s$ develop non-zero VEVs. Denoting 
$\langle h\rangle=v,\langle s\rangle=u$, extremization of the low energy scalar potential (2) requires

$$
\begin{aligned}
& v^{2}=2 \frac{\lambda_{h s} m_{s}^{2}-2 \lambda_{s} m_{h}^{2}}{4 \lambda_{s} \lambda_{h}-\lambda_{h s}^{2}}, \\
& u^{2}=2 \frac{\lambda_{h s} m_{h}^{2}-2 \lambda_{h} m_{s}^{2}}{4 \lambda_{s} \lambda_{h}-\lambda_{h s}^{2}} .
\end{aligned}
$$

The diagonal matrix elements of the Hessian at this point are $2 \lambda_{s} u^{2}$ and $2 \lambda_{h} v^{2}$, while its determinant is $\left(4 \lambda_{s} \lambda_{h}-\lambda_{h s}^{2}\right) v^{2} u^{2}$. Then, the extremum is a local minimum if

$$
\begin{aligned}
& \lambda_{h s} m_{h}^{2}-2 \lambda_{h} m_{s}^{2}>0, \\
& \lambda_{h s} m_{s}^{2}-2 \lambda_{s} m_{h}^{2}>0, \\
& 4 \lambda_{s} \lambda_{h}-\lambda_{h s}^{2}>0 .
\end{aligned}
$$

In this case, the mass squared eigenvalues are

$$
m_{1,2}^{2}=\lambda_{h} v^{2}+\lambda_{s} u^{2} \mp \sqrt{\left(\lambda_{s} u^{2}-\lambda_{h} v^{2}\right)^{2}+\lambda_{h s}^{2} u^{2} v^{2}}
$$

with the mixing angle $\theta$ given by

$$
\tan 2 \theta=\frac{\lambda_{h s} u v}{\lambda_{h} v^{2}-\lambda_{s} u^{2}} .
$$

Following the convention of [16], the mixing angle is defined by

$$
O^{T} M^{2} O=\operatorname{diag}\left(m_{1}^{2}, m_{2}^{2}\right) \quad, \quad O=\left(\begin{array}{cc}
\cos \theta & \sin \theta \\
-\sin \theta & \cos \theta
\end{array}\right),
$$

where $M^{2}$ is a $2 \times 2$ mass squared matrix. The range of $\theta$ is related to the ordering of the eigenvalues through $\operatorname{sign}\left(m_{1}^{2}-m_{2}^{2}\right)=\operatorname{sign}\left(\lambda_{s} u^{2}-\lambda_{h} v^{2}\right) \operatorname{sign}(\cos 2 \theta)$ and we take $m_{1}$ to be the smaller eigenvalue. The mass eigenstates are

$$
\begin{aligned}
& H_{1}=s \cos \theta-h \sin \theta, \\
& H_{2}=s \sin \theta+h \cos \theta .
\end{aligned}
$$

Note that the lighter mass eigenstate $H_{1}$ is "Higgs-like" for $\lambda_{s} u^{2}>\lambda_{h} v^{2}$ and "singlet-like" otherwise. The former case corresponds to $|\theta|>\pi / 4$.

\subsection{Large singlet VEV limit}

In the limit $u \gg v$, we have

$$
\begin{aligned}
& m_{1}^{2} \simeq 2\left(\lambda_{h}-\frac{\lambda_{h s}^{2}}{4 \lambda_{s}}\right) v^{2}, \\
& m_{2}^{2} \simeq 2 \lambda_{s} u^{2}+\frac{\lambda_{h s}^{2}}{2 \lambda_{s}} v^{2}, \\
& \tan 2 \theta \simeq-\frac{\lambda_{h s} v}{\lambda_{s} u},
\end{aligned}
$$


where the neglected terms are suppressed by $v^{2} / u^{2}$. As $u$ increases, $\tan 2 \theta \rightarrow 0$ and the singletHiggs mixing approaches zero. The light eigenstate $H_{1}$ is almost a pure Higgs, yet its mass squared is not $2 \lambda_{h} v^{2}$ as in the $\mathrm{SM}$, but receives a finite negative correction $-\lambda_{h s}^{2} v^{2} /\left(2 \lambda_{s}\right)$. Therefore, a given Higgs mass corresponds to a larger $\lambda_{h}$ than what it would be in the Standard Mode1. A $125 \mathrm{GeV}$ Higgs can then be obtained for various $\lambda_{h}$ up to order one as long as

$$
\lambda_{h}-\frac{\lambda_{h s}^{2}}{4 \lambda_{s}} \simeq 0.13
$$

As a result, all the couplings can remain positive at all scales, ensuring stability of the potential. This can be achieved with tiny $\lambda_{h s}$ and $\lambda_{s}$ as long as $\lambda_{h s}^{2} / \lambda_{s}$ is significant.

We note that although the heavy eigenstate decouples for $u \rightarrow \infty$, the effect on the light eigenvalue remains finite. This limit does not correspond to simply sending the heavy mass to infinity, but also involves changing the vacuum state. The SM vacuum is not recovered due to the large cross term $\lambda_{h s} u^{2} v^{2}$, although the light state is (almost) exactly SM-like. The cross term generates a leading order $\mathcal{O}\left(u^{2} v^{2}\right)$ correction to the determinant of the mass squared matrix, resulting in a finite shift in $m_{1}$.

Rewriting

$$
\begin{aligned}
& m_{h}^{2}=-\frac{1}{2} \lambda_{h s} u^{2}-\lambda_{h} v^{2} \\
& m_{s}^{2}=-\frac{1}{2} \lambda_{h s} v^{2}-\lambda_{s} u^{2}
\end{aligned}
$$

one finds that the $u \rightarrow \infty$ limit means that both $m_{s}^{2}$ and $m_{h}^{2}$ increase in magnitude indefinitely, while $m_{s}^{2} / m_{h}^{2} \rightarrow 2 \lambda_{s} / \lambda_{h s}$. The hierarchy between $v$ and $u$ is then equivalent to tuning of $m_{s}^{2} / m_{h}^{2}$ to this value. Note also that the vacuum energy is negative and scales like $-u^{4}$.

Since the singlet-Higgs mixing can be very small, this scenario is essentially indistinguishable from the SM apart from the Higgs quartic coupling. Suppose that both mass eigenstates are sufficiently light so that they are degrees of freedom of our TeV-scale effective theory, although $m_{2} \gg m_{1}$. Given the Higgs mass $m_{\text {Higgs }}$ (which we also identify with $m_{1}$ ), the quartic couplings are given by

$$
\begin{aligned}
\left.\lambda_{h}\right|_{\mathrm{SM}} & =\frac{m_{\text {Higgs }}^{2}}{2 v^{2}}, \\
\left.\lambda_{h}\right|_{\mathrm{SM}+\text { singlet }} & =\frac{m_{\text {Higgs }}^{2}}{2 v^{2}}+\frac{\lambda_{h s}^{2}}{4 \lambda_{s}} .
\end{aligned}
$$

\footnotetext{
${ }^{1}$ This phenomenon does not occur in conventional 2 Higgs doublet models since the field VEVs are bounded by $246 \mathrm{GeV}$ in that case.
} 
For a $\mathrm{TeV}$-scale singlet, the correction to the quartic Higgs coupling can be of order $100 \%$, whereas the gauge-Higgs coupling is modified by $10 \%$. An ILC [20] or high-luminosity LHC [21] study of the Higgs self-coupling would then be able to reveal the presence of the singlet. On the other hand, if the singlet is very heavy, the effective low energy theory contains just the Higgs doublet and the Higgs mass-coupling relation is not affected [22]. The quartic coupling receives the singlet-induced contribution above the singlet threshold, which has an analogous stabilizing effect.

\section{$2.3 \quad$ Numerical example}

The quartic couplings at high energies are determined by the following 1-loop renormalization group equations (see e.g. [23])

$$
\begin{aligned}
16 \pi^{2} \frac{d \lambda_{h}}{d t} & =24 \lambda_{h}^{2}-6 y_{t}^{4}+\frac{3}{8}\left(2 g^{4}+\left(g^{2}+g^{\prime 2}\right)^{2}\right) \\
& +\left(-9 g^{2}-3 g^{\prime 2}+12 y_{t}^{2}\right) \lambda_{h}+\frac{1}{2} \lambda_{h s}^{2}, \\
16 \pi^{2} \frac{d \lambda_{h s}}{d t} & =4 \lambda_{h s}^{2}+12 \lambda_{h} \lambda_{h s}-\frac{3}{2}\left(3 g^{2}+g^{\prime 2}\right) \lambda_{h s} \\
& +6 y_{t}^{2} \lambda_{h s}+6 \lambda_{s} \lambda_{h s}, \\
16 \pi^{2} \frac{d \lambda_{s}}{d t} & =2 \lambda_{h s}^{2}+18 \lambda_{s}^{2},
\end{aligned}
$$

where $t=\ln \left(\mu / m_{t}\right)$ with $\mu$ being the energy scale. The effect of the heavy singlet threshold can be included by inserting appropriate Heaviside $\theta$-functions. The RG equations for the gauge and the top Yukawa couplings are given by the usual SM expressions. The low energy input values for these couplings are $g\left(m_{t}\right)=0.64, g^{\prime}\left(m_{t}\right)=0.35, g_{3}\left(m_{t}\right)=1.16$, while for the top Yukawa coupling we use its running value at $m_{t}, y_{t}\left(m_{t}\right)=0.93$ [24]. A two-loop SM running of $\lambda_{h}$ as well as finite corrections are considered in a recent analysis [12] (see [25] for a discussion). Within the uncertainties of $m_{t}$, our 1-loop SM results are consistent with theirs.

Figure 1 displays a few possible choices of low energy couplings consistent with $m_{h} \simeq 125$ $\mathrm{GeV}$ and the resulting evolution of $\lambda_{h}$ with energy. We have chosen small couplings $\sim 10^{-2}$ in which case the singlet loop contribution to $\lambda_{h}$ is suppressed, as is the singlet threshold effect (for definiteness, we have set the singlet mass to be sub-TeV). For $\lambda_{h s}^{2} /\left(4 \lambda_{s}\right)$ greater than about 0.015, the Higgs quartic coupling is positive at all scales. As seen from the RG equations, $\lambda_{s}$ remains positive if it is positive at low energies. This also applies to $\lambda_{h s}$ as its evolution is dominated by the top-quark contribution. The potential is therefore positive-definite at high 


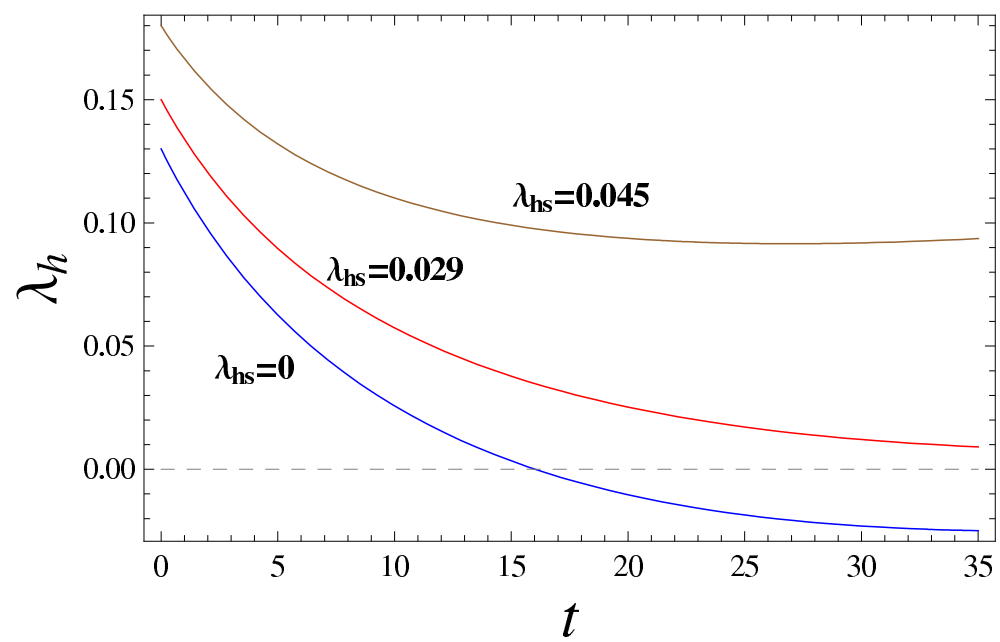

Figure 1: Higgs quartic coupling evolution with energy for various $\lambda_{h s}$ and $\lambda_{s}=0.01$ at $m_{t}$. The Higgs mass is fixed at approximately $125 \mathrm{GeV}$.

energies and the EW vacuum is stable. Note that this result can be achieved with tiny $\lambda_{h s}$ and $\lambda_{s}$ since what matters is $\lambda_{h s}^{2} /\left(4 \lambda_{s}\right)$.

Analogous examples can be constructed for $\lambda_{h s}<0$ as well. In that case, one must obey the additional constraint $4 \lambda_{s} \lambda_{h}-\lambda_{h s}^{2}>0$ at all scales. Even though this quantity is positive in the low energy limit, it may turn negative at higher energies signifying the existence of a run-away direction.

This illustrates how the electroweak vacuum can be stabilized by invoking a heavy scalar which has no observable effects other than changing the Higgs self-coupling. In that case, the bound on the reheating temperature [12] relaxes and, furthermore, the Higgs field can be responsible for inflation [5].

One may also stabilize the Higgs potential entirely by loop effects involving a relatively light singlet with a zero VEV [26, 27, 28]. As seen from the RG equations, the singlet makes a positive contribution to $\lambda_{h}$, so a substantial cross coupling $\lambda_{h s} \sim 0.4$ would stabilize the electroweak vacuum. Although viable, this mechanism is quite constraining and the options for Higgs-like inflation would be limited. In contrast, the tree-level mechanism described above works even for very small couplings and a heavy singlet providing ample opportunities for model building. 


\section{Rescuing Higgs inflation}

Scalar fields with the usual quartic potentials can drive inflation if they possess large couplings $\xi_{i} \gg 1$ to scalar curvature $R$ [5, 29]. Below we summarize basic ideas of Higgs portal inflation, closely following Ref. [16]. Variants of this scenario which include dark matter considerations have appeared in [23, 30].

\subsection{The setup}

The relevant Jordan frame Lagrangian in the unitary gauge is

$$
\mathcal{L} / \sqrt{-g}=-\frac{1}{2} M_{\mathrm{Pl}}^{2} R-\frac{1}{2} \xi_{h} h^{2} R-\frac{1}{2} \xi_{s} s^{2} R+\frac{1}{2}\left(\partial_{\mu} h\right)^{2}+\frac{1}{2}\left(\partial_{\mu} s\right)^{2}-V
$$

with $\xi_{h, s}>0$. The scalar couplings to curvature can be eliminated by a conformal transformation

$$
\tilde{g}_{\mu \nu}=\Omega^{2} g_{\mu \nu}, \Omega^{2}=1+\frac{\xi_{h} h^{2}+\xi_{s} s^{2}}{M_{\mathrm{Pl}}^{2}},
$$

which brings us to the Einstein frame. Let us set $M_{\mathrm{Pl}}$ to 1 and consider the limit

$$
\xi_{h} h^{2}+\xi_{s} s^{2} \gg 1
$$

At $\xi \equiv \xi_{h}+\xi_{s} \gg 1$, the kinetic terms and the potential for new variables

$$
\begin{aligned}
\chi & =\sqrt{\frac{3}{2}} \log \left(\xi_{h} h^{2}+\xi_{s} s^{2}\right), \\
\tau & =\frac{h}{s}
\end{aligned}
$$

are given by

$$
\mathcal{L}_{\text {kin }}=\frac{1}{2}\left(\partial_{\mu} \chi\right)^{2}+\frac{1}{2} \frac{\xi_{h}^{2} \tau^{2}+\xi_{s}^{2}}{\left(\xi_{h} \tau^{2}+\xi_{s}\right)^{3}}\left(\partial_{\mu} \tau\right)^{2}
$$

and

$$
U=\frac{\lambda_{h} \tau^{4}+\lambda_{h s} \tau^{2}+\lambda_{s}}{4\left(\xi_{h} \tau^{2}+\xi_{s}\right)^{2}}
$$

respectively. Note that at large $\chi$, the potential is independent of $\chi$ which allows for slow-roll inflation. The composition of the inflaton depends on the value of $\tau$ at the minimum of the potential. The minima of $U$ are classified as follows

$$
\begin{array}{ll}
\text { (1) } 2 \lambda_{h} \xi_{s}-\lambda_{h s} \xi_{h}>0,2 \lambda_{s} \xi_{h}-\lambda_{h s} \xi_{s}>0, & \tau=\sqrt{\frac{2 \lambda_{s} \xi_{h}-\lambda_{h s} \xi_{s}}{2 \lambda_{h} \xi_{s}-\lambda_{h s} \xi_{h}}} \\
\text { (2) } 2 \lambda_{h} \xi_{s}-\lambda_{h s} \xi_{h}>0,2 \lambda_{s} \xi_{h}-\lambda_{h s} \xi_{s}<0, & \tau=0 \\
\text { (3) } 2 \lambda_{h} \xi_{s}-\lambda_{h s} \xi_{h}<0,2 \lambda_{s} \xi_{h}-\lambda_{h s} \xi_{s}>0, & \tau=\infty \\
\text { (4) } 2 \lambda_{h} \xi_{s}-\lambda_{h s} \xi_{h}<0,2 \lambda_{s} \xi_{h}-\lambda_{h s} \xi_{s}<0, & \tau=0, \infty
\end{array}
$$



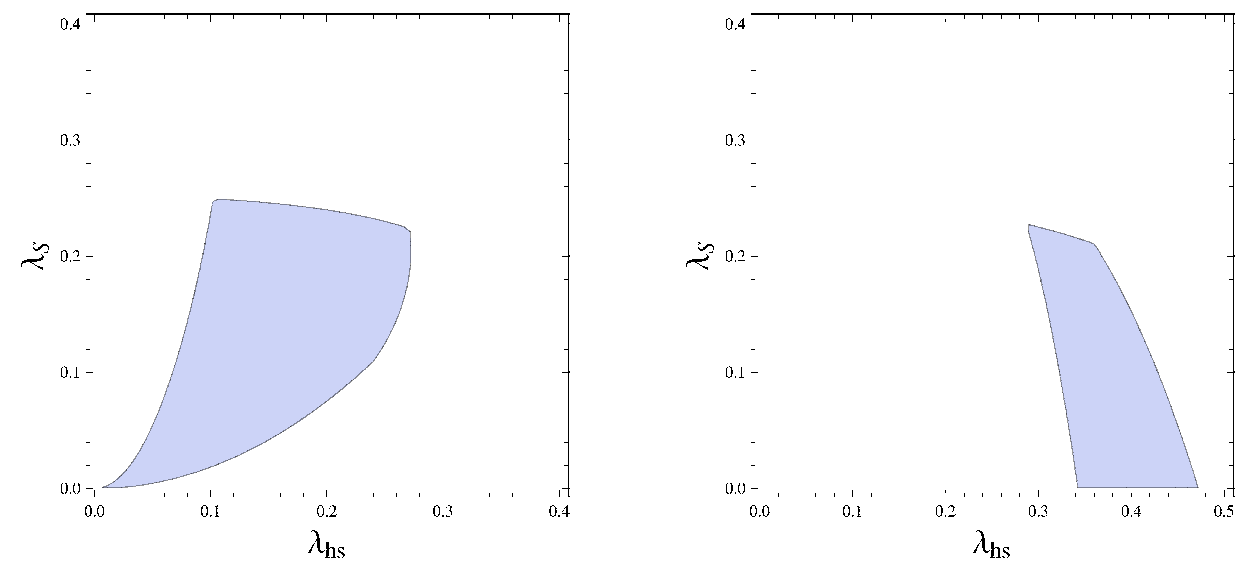

Figure 2: Parameter space allowed by Higgs inflation for the two vacua: $u \gg v$ (left) and $u=0$ (right). The Higgs mass is fixed at approximately $125 \mathrm{GeV}$ and $\xi_{s} / \xi_{h}=10^{-3}$.

In all of these minima, the $\tau$ field is heavy $(m \sim 1 / \sqrt{\xi}$ in Planck units) and can be integrated out, leaving $\chi$ as the only dynamical variable during inflation. If we are to retain the subleading $M_{\mathrm{Pl}}^{2} /\left(\xi_{h} h^{2}+\xi_{s} s^{2}\right)$ term in $\Omega^{2}$, the potential for $\chi$ becomes

$$
U(\chi)=\frac{\lambda_{\text {eff }}}{4 \xi_{h}^{2}}\left(1+\exp \left(-\frac{2 \chi}{\sqrt{6}}\right)\right)^{-2}
$$

in Planck units. Here $\lambda_{\text {eff }}$ depends on the composition of the inflaton. For Higgs inflation, $\lambda_{\text {eff }}=\lambda_{h}$; for singlet inflation, $\lambda_{\text {eff }}=\lambda_{s} / x^{2}$ with $x=\xi_{s} / \xi_{h}$; and for mixed inflation, $\lambda_{\text {eff }}=$ $\left(4 \lambda_{s} \lambda_{h}-\lambda_{h s}^{2}\right) /\left[4\left(\lambda_{s}+\lambda_{h} x^{2}-\lambda_{h s} x\right)\right]$.

Depending on the values of $\lambda_{i}$ and $\xi_{i}, 3$ variants of inflation are possible. Since the shape of the potential is the same in all cases (at tree level), they all share the same prediction for the spectral index $n \simeq 1-2 / N$ and the tensor to scalar perturbation ratio $r \simeq 12 / N^{2}$, with $N$ being the number of $e$-folds during inflation [5]. Obviously, the prerequisite for inflation is that the relevant $\lambda_{\text {eff }}$ be positive at high energies. Then, given the low value of the Higgs mass, Higgs inflation becomes problematic [31, 32, 33]. To revive this option, one may use the mechanism of $\lambda_{h}$-enhancement described in the previous section.

\subsection{Singlet-assisted Higgs inflation}

Although the minimalistic version of the original Higgs inflation [5] appears disfavored, the presence of an almost decoupled singlet can save the idea. Of course, the Higgs field is no longer special in this case as there is another scalar which could drive inflation. Yet, Higgs inflation remains an interesting possibility. 
For Higgs inflation to occur, we require the following conditions at the Hubble scale $\mu_{H} \sim$ $M_{\mathrm{Pl}} / \xi_{h}$ with $\xi_{h} \sim 5 \times 10^{4}[5]:$

$$
\begin{aligned}
& \lambda_{h}>0, \\
& 2 \lambda_{h} \frac{\xi_{s}}{\xi_{h}}-\lambda_{h s}<0 .
\end{aligned}
$$

The second constraint follows from (20) and ensures that $\tau=\infty$ is a local minimum. (The sign of $2 \lambda_{s} \xi_{h}-\lambda_{h s} \xi_{s}$ determines whether this is the only local minimum.) It follows then that $\lambda_{h s}$ must be positive, $\lambda_{h s}>0$. Furthermore, we impose the "perturbativity" constraint

$$
\left|\lambda_{i}\right|<1
$$

at the Hubble scale. This judicious choice is motivated by perturbativity $\left(\lambda_{i}^{2} /(4 \pi)<1\right)$ all the way up to the Planck scale.

We take a conservative viewpoint [16] and do not impose further constraints arising from loop corrections to the shape of the inflaton potential (the "running" spectral index). These are likely to be affected by the presence of higher dimensional operators and/or heavy states [34, 35, 36, 37, 38. In the absence of a UV complete theory, such subtle effects cannot be calculated reliably.

The allowed parameter space for $\xi_{h} \gg \xi_{s}$ is shown in Fig. 2, Here we set the singlet mass in the $\mathrm{TeV}$ range, so the threshold effects are unimportant. The left panel corresponds to the $u \gg v$ vacuum such that $\lambda_{h}-\lambda_{h s}^{2} /\left(4 \lambda_{s}\right) \simeq 0.13$ at the EW scale. The right panel corresponds to $u=0$ and $\lambda_{h} \simeq 0.13$ at the EW scale, in which case the stabilization is due to loop effects. The obvious difference is that the first option allows for arbitrarily small couplings $\lambda_{h s}, \lambda_{s}$, whereas in the other case $\lambda_{h s}$ must be greater than 0.3-0.4. The allowed region is bounded on the right and from above by perturbativity, and on the left by $\lambda_{h}\left(\mu_{H}\right)>0$ in both cases. The constraint $2 \lambda_{h} \xi_{s} / \xi_{h}-\lambda_{h s}<0$ amounts essentially to positivity of $\lambda_{h s}$. Finally, we note that the magnitude of $\xi_{h} \sim 5 \times 10^{4}$ is fixed by the potential normalization [5].

The two possibilities can potentially be distinguished at colliders. The $u \gg v$ option can lead to a significant correction to the Higgs self-coupling $\lambda_{h}$, whereas the $u=0$ case requires a substantial Higgs coupling to the singlet. To observe production of the (EW-scale) singlet pairs would be challenging, but feasible given very high luminosities [18].

Finally, we note that other variants of Higgs portal inflation are possible. The inflaton can also be a mixture of the Higgs and the singlet as well as the singlet alone, depending on $\lambda_{i}$ and 
$\xi_{i}$ [16]. To disentangle all the possibilities at colliders would be very challenging as it requires determination of the sign of $\lambda_{h s}$.

\section{Conclusion}

We have studied the possibility that the Higgs boson has a small admixture of an SM singlet. We find that, as the singlet VEV increases, the Higgs mass recieves a tree level contribution which does not vanish in the zero-mixing/heavy-singlet limit. Such a correction can be order one and make the EW vacuum completely stable rather than metastable. The requisite Higgs-singlet coupling $\lambda_{h s}$ and the singlet self-coupling $\lambda_{s}$ are allowed to be arbitrarily small (in practical terms), as long as $\lambda_{h s}^{2} /\left(4 \lambda_{s}\right)$ is greater than about 0.015 for a TeV-scale singlet. This situation is practically indistinguishable from the SM at low energies unless the Higgs quartic coupling is measured.

We also find that Higgs inflation is possible in our framework since the quartic coupling remains positive at high energies. This result can also be achieved purely through singletinduced loop effects, although a substantial Higgs-singlet coupling would be required in this case.

Acknowledgements. The author is grateful to H.M. Lee for useful comments.

\section{References}

[1] V. Silveira and A. Zee, Phys. Lett. B 161, 136 (1985).

[2] In the context of mirror matter, the Higgs portal interaction also appeared in R. Foot, H. Lew and R. R. Volkas, Phys. Lett. B 272, 67 (1991).

[3] S. Kanemura, S. Matsumoto, T. Nabeshima and N. Okada, Phys. Rev. D 82, 055026 (2010).

[4] O. Lebedev, H. M. Lee and Y. Mambrini, Phys. Lett. B 707, 570 (2012).

[5] F. L. Bezrukov and M. Shaposhnikov, Phys. Lett. B 659, 703 (2008).

[6] R. Schabinger and J. D. Wells, Phys. Rev. D 72, 093007 (2005).

[7] B. Patt and F. Wilczek, arXiv:hep-ph/0605188.

[8] J. McDonald, Phys. Rev. D50 (1994) 3637-3649. 
[9] C. P. Burgess, M. Pospelov, T. ter Veldhuis, Nucl. Phys. B619 (2001) 709-728.

[10] V. Barger, P. Langacker, M. McCaskey, M. J. Ramsey-Musolf and G. Shaughnessy, Phys. Rev. D 77, 035005 (2008).

[11] C. Englert, T. Plehn, D. Zerwas and P. M. Zerwas, Phys. Lett. B 703, 298 (2011); C. Englert, T. Plehn, M. Rauch, D. Zerwas and P. M. Zerwas, Phys. Lett. B 707, 512 (2012).

[12] J. Elias-Miro, J. R. Espinosa, G. F. Giudice, G. Isidori, A. Riotto and A. Strumia, arXiv:1112.3022 [hep-ph].

[13] J. R. Espinosa, G. F. Giudice and A. Riotto, JCAP 0805, 002 (2008).

[14] ATLAS Collaboration, arXiv:1202.1408 [hep-ex].

[15] S. Chatrchyan et al. [CMS Collaboration], arXiv:1202.1488 [hep-ex].

[16] O. Lebedev and H. M. Lee, Eur. Phys. J. C 71, 1821 (2011).

[17] M. Bowen, Y. Cui and J. D. Wells, JHEP 0703, 036 (2007); I. Low, P. Schwaller, G. Shaughnessy and C. E. M. Wagner, Phys. Rev. D 85, 015009 (2012); B. Batell, S. Gori and L. -T. Wang, arXiv:1112.5180 [hep-ph].

[18] A. Djouadi, O. Lebedev, Y. Mambrini and J. Quevillon, arXiv:1112.3299 [hep-ph]; X. G. He, B. Ren and J. Tandean, arXiv:1112.6364 [hep-ph]; J. F. Kamenik and C. Smith, arXiv:1201.4814 [hep-ph].

[19] Y. Mambrini, Phys. Rev. D 84, 115017 (2011); S. Andreas, T. Hambye and M. H. G. Tytgat, JCAP 0810, 034 (2008).

[20] G. J. Gounaris, D. Schildknecht and F. M. Renard, Phys. Lett. B 83, 191 (1979); V. D. Barger, T. Han and R. J. N. Phillips, Phys. Rev. D 38, 2766 (1988); A. Djouadi, W. Kilian, M. Muhlleitner and P. M. Zerwas, Eur. Phys. J. C 10, 27 (1999).

[21] U. Baur, T. Plehn and D. L. Rainwater, Phys. Rev. D 69, 053004 (2004).

[22] J. Elias-Miro, Jose, R. Espinosa, G. F. Giudice, H. M. Lee and A. Strumia, arXiv:1203.0237 [hep-ph].

[23] R. N. Lerner and J. McDonald, Phys. Rev. D 80 (2009) 123507. 
[24] U. Langenfeld, S. O. Moch and P. Uwer, arXiv:1006.0097.

[25] M. Holthausen, K. S. Lim and M. Lindner, JHEP 1202, 037 (2012).

[26] M. Gonderinger, Y. Li, H. Patel and M. J. Ramsey-Musolf, JHEP 1001, 053 (2010).

[27] M. Kadastik, K. Kannike, A. Racioppi and M. Raidal, arXiv:1112.3647 [hep-ph].

[28] C. S. Chen and Y. Tang, arXiv:1202.5717 [hep-ph].

[29] F. Bezrukov, A. Magnin, M. Shaposhnikov and S. Sibiryakov, JHEP 1101, 016 (2011).

[30] T. E. Clark, B. Liu, S. T. Love and T. ter Veldhuis, Phys. Rev. D 80, 075019 (2009).

[31] F. L. Bezrukov, A. Magnin and M. Shaposhnikov, Phys. Lett. B 675, 88 (2009);

F. Bezrukov and M. Shaposhnikov, JHEP 0907, 089 (2009).

[32] A. De Simone, M. P. Hertzberg and F. Wilczek, Phys. Lett. B 678, 1 (2009).

[33] A. O. Barvinsky, A. Y. Kamenshchik, C. Kiefer, A. A. Starobinsky and C. F. Steinwachs, arXiv:0910.1041 [hep-ph].

[34] C. P. Burgess, H. M. Lee and M. Trott, JHEP 0909, 103 (2009); C. P. Burgess, H. M. Lee and M. Trott, JHEP 1007, 007 (2010).

[35] J. L. F. Barbon and J. R. Espinosa, Phys. Rev. D 79, 081302 (2009).

[36] R. N. Lerner and J. McDonald, Phys. Rev. D 82, 103525 (2010).

[37] G. F. Giudice and H. M. Lee, Phys. Lett. B 694, 294 (2011).

[38] M. P. Hertzberg, arXiv:1110.5650 [hep-ph]. 